\title{
Technetium Tc 99 \\ Hydrazinonicotinamide-Tricine-linked Interleukin-2
}

National Cancer Institute

\section{Source}

National Cancer Institute. Technetium Tc 99 Hydrazinonicotinamide-Tricine-linked

Interleukin-2. NCI Thesaurus. Code C104731.

A radioconjug ate composed of the cytokine interleukin-2 (IL-2) conjug ated to the bifunctional chelating agent succinimidyl-6-hydrazinopyridine-3-carboxylate (HYNIC-NHS) and co-ligand tricine and labeled with the radioisotope technetium Tc 99m (99mT cHYNIC-IL2), that can potentially be used as a diagnostic agent to detect tumor inflammation. Upon intravenous administration, IL-2 selectively binds to the IL-2 receptor (IL-2R) expressed on tumor infiltrating lymphocytes (TILs); upon internalization, the Tc99m moiety can be visualized using radioimaging, which allows the quantification of TILs expressing IL-2R. This agent detects tumor enlargement due to invasion by TILS and not tumor progression. 